\title{
LINEAR DISTRIBUTION PROCESSES
}

\author{
L. BEL, G. OPPENHEIM, L. ROBBIANO, and M.C. VIANO \\ Equipe de modélisation, stochastique et statistique \\ Bât 425, Université de Paris Sud \\ 91405 Orsay Cedex, France \\ E-mail: Liliane.Bel@math.u-psud.fr
}

(Received May, 1996; Revised June, 1997)

In this paper, we propose a generalization of continuous-time processed defined by

$$
X_{t}=\int f(t-s) d W_{s}
$$

to the case of $f$ being a distribution. We give a necessary and sufficient condition for $f$, such that the obtained process is a second order distribution process. We study the moments and the regularity of these processes. In addition, we investigate a generalization to processes with stationary increments.

Key words: Distribution Processes, Sobolev Spaces, Regularity, Stationary, Increments.

AMS subject classifications: $60 \mathrm{G} 20,60 \mathrm{G} 17,60 \mathrm{G} 10,46 \mathrm{E} 35$.

\section{Introduction}

Here we study linear models defined by a convolution of the form

$$
X_{t}=\int f(t-s) d W_{s}
$$

where $f$ is in the space $\mathscr{I}^{\prime}$ of distributions. These processes are distribution processes, since they are indexed by test functions. This situation generalizes the familiar setup, where $f$ is in $L^{2}$. The induced process $X_{t}$ is then a second order stationary linear process.

The most popular example of distribution processes is probably the derivative of the Brownian motion. Selecting $f$ in (1) from a suitable family provides interesting families of distribution processes. For instance, in [1] we study the particular family of fractional ARMA distribution processes.

More generally, many field like Physics or Statistical Mechanics often need a 
larger framework than the time indexed stochastic processes one. Distribution processes have been independently introduced by Gelfand and Vilenkin [8] and K. Itô [11]. These processes were studied afterwards from various points of view: Fernique [7] showed that distribution processes are random distributions and investigated their general properties (characteristic functions, moments, convergence in law and pointwise convergence), Meidan $([16,17])$ studied the Reproducing Kernel Hilbert Space of distribution processes and established the connection between time indexed stochastic processes and distribution processes. Esquivel [5] dealt with periodic random distributions and their Fourier coefficients. Rao [21] gave a characterization of harmonizable distribution processes. Piterbarg [19] investigated the structure of the $\sigma$-algebra generated by a distribution process with rational spectrum. Brillinger [2] proved the asymptotic normality for finite Fourier transforms of stationary distribution processes.

Other authors (Inaba et al. [10], Lozanov et al. [14], Y. Itô [12]), studied processes on different function spaces such as $L^{2}$ or hyperfunctions. Pugh [20] used the $L^{2}$ processes framework to study the analytic signal of white noise.

The usually adopted terminology is somewhat confusing: distribution processes, that is, processes indexed by the set of test functions, are often called generalized processes. Unfortunately, the same term is used for time indexed processes with range in a distribution space, which are widely used in white noise calculus [13], functionals of Brownian motions $[9,18]$ or branching random fields [6].

For this reason, we prefer to call our processes distribution processes.

We first recall the main properties and the definition of regularity of distribution processes, using the Gaussian feature allowing techniques of functional analysis. We then show that expression (1) makes sense if and only if the distribution $f$ belongs to the Sobolev space $H^{-\infty}$.

The induced Gaussian linear stationary processes are indexed by test functions. As Gaussian linear stationary processes time indexed, they have a spectral density and we give the expression for their moments. We give the link between the regularity of distribution processes $X$ and of the corresponding filters $f$ : namely, $X$ is of Hölder regularity $s$ if and only if $f$ belongs to the Besov space $B_{2, \infty}^{s}$.

We carry out an analogous study on distribution processes with stationary increments that generalize continuous-time processes parametrized by functions $f$ and $g$, of the form

$$
X_{t}=\int(f(t-s)-g(-s)) d W_{s}
$$

where $f$ and $g$ are distributions.

This family contains the popular fractional Brownian motion and the family described previously.

\section{Second-Order Distribution Processes}

We recall in this section the definition and properties of second-order distribution processes. These processes form a subclass of those introduced by Gelfand and Vilenkin [8]. Most of following are results from [8] and [11]. 


\subsection{Definition and properties of distribution processes}

Let $\mathcal{C}_{0}^{\infty}\left(\mathbb{R}^{n}\right)$ denote the space of infinitely differentiable functions with compact support in $\mathbb{R}^{n}$, with the family of semi-norms

$$
\|\varphi\|_{k}=\sum_{\substack{\gamma \in \mathbb{N}^{n} \\|\gamma| \leq k}}\left\|\partial^{\gamma} \varphi\right\|_{L^{\infty}\left(\mathbb{R}^{n}\right)^{.}}
$$

Let $(\Omega, \mathcal{A}, P)$ denote a fixed probability space and $L^{2}(\Omega)$ the space of second-order random variables. In the sequel, $C$ will denote a constant.

Definition 1: A second-order distribution process $X$ defined on the probability space $(\Omega, \mathcal{A}, P)$ is a continuous application from the space $\mathcal{C}_{0}^{\infty}\left(\mathbb{R}^{n}\right)$ into $L^{2}(\Omega)$ :

$$
\text { for } \varphi \in \mathbb{C}_{0}^{\infty}\left(\mathbb{R}^{n}\right), \quad X(\varphi):=\langle X ; \varphi\rangle \in L^{2}(\Omega),
$$

and for every compact subset $K$ in $\mathbb{R}^{n}$,

$$
\exists C_{K}, \exists k, \forall \varphi \in \mathcal{C}_{0}^{\infty}(K): \quad\|\langle X ; \varphi\rangle\|_{L^{2}(\Omega)} \leq C_{K}\|\varphi\|_{k}
$$

This definition, that requires the mean-square continuity of the application $X$, is more suitable for our purpose than that of [8], which requires only convergence-in-law continuity. As we consider only second-order distribution processes, for the sake of simplicity and by a slight abuse of terminology, we will henceforth simply refer to them as distribution processes. The space of distribution processes will be denoted by $\mathscr{\Phi}^{\prime}\left(\mathbb{R}^{n}, L^{2}(\Omega)\right)$ by analogy with the space of distributions $\mathscr{\Phi}^{\prime}\left(\mathbb{R}^{n}\right)$.

Meidan [16] showed that a continuous-time process with values in $L^{2}(\Omega)$ is a distribution process if and only if it is locally Bochner integrable, that is if $\left\|X_{t}\right\|_{L^{2}(\Omega)} \in L_{l o c}^{2}\left(\mathbb{R}^{n}\right)$. In this case, we set:

$$
\forall \varphi \in \mathrm{C}_{0}^{\infty}\left(\mathbb{R}^{n}\right),\langle X ; \varphi\rangle=\int X_{t} \varphi(t) d t .
$$

Fernique [7] showed that these processes can be seen as random distributions. Namely, if $X$ is a distribution process, then there exists a measurable function $X_{1}$ from $\Omega$ into $\mathscr{D}^{\prime}\left(\mathbb{R}^{n}\right)$ such that for all $\varphi \in \mathbb{C}_{0}^{\infty}\left(\mathbb{R}^{n}\right)$ and for almost all $\omega$,

$$
\left\langle X_{1}(\omega) ; \varphi\right\rangle=\langle X ; \varphi\rangle(\omega)
$$

Let $\mathscr{K}\left(\mathbb{R}^{n}\right)$ be a subset of distributions and $\mathscr{G}\left(\mathbb{R}^{n}, L^{2}(\Omega)\right)$ the set of distribution processes $X$ in $\mathscr{D}^{\prime}\left(\mathbb{R}^{n}, L^{2}(\Omega)\right)$, such that $X(\omega) \in \mathfrak{O}\left(\mathbb{R}^{n}\right)$ for almost all $\omega$.

We define the derivation, translation, convolution, and Fourier transform (denoted by $\mathscr{F}(T)$ or $\widehat{T}$ ) for distribution processes in the same way as for distributions. We also define the mean, covariance and stationarity of these processes in the same way as for continuous processes. In particular, we have the following properties:

Property 1: ([8]) Let $X$ be a second order stationary distribution process. The mean $m$ of $X$ satisfies:

$$
\exists a \in \mathbb{C}, \quad \forall \varphi \in \mathcal{C}_{0}^{\infty}\left(\mathbb{R}^{n}\right): \quad m(\varphi)=\int a \varphi(t) d t,
$$

and there exists a positive tempered measure $\mu$ such that the covariance $B$ of $X$ has 
the form

$$
B(\varphi, \psi)=\int \widehat{\varphi}(\xi) \overline{\widehat{\psi}(\xi)} d \mu(\xi)
$$

$\mu$ is called the spectral measure of $X$. If $\mu$ admits a density $g$ with respect to the Lebesgue measure, then $g$ is called the spectral density of $X$.

Property 2: ([11]) If $X$ is a second order stationary distribution process with spectral measure $\mu$, then there exists a random measure $M$ with respect to $\mu$, such that

$$
\langle X ; \varphi\rangle=\int \widehat{\varphi} d M(\lambda)=M(\widehat{\varphi})
$$

Conversely, any distribution process having such a form is a second order stationary distribution process.

\subsection{Regularity properties}

We now define regularity for distribution processes by characterizing the spaces they belong to in the same way as for distribution spaces. Firstly, we recall some definitions and properties of different distribution spaces [24].

Let $\varphi\left(\mathbb{R}^{n}\right)$ denote the space of $\mathcal{C}^{\infty}$, rapidly decreasing functions and let $\varphi^{\prime}\left(\mathbb{R}^{n}\right)$ be its dual, space of tempered distributions [22].

Denote $\langle\xi\rangle^{2}=1+|\xi|^{2}$. The Sobolev space $H^{s}\left(\mathbb{R}^{n}\right), s \in \mathbb{R}$, is defined by

$$
H^{s}\left(\mathbb{R}^{n}\right)=\left\{f \in \varphi^{\prime}\left(\mathbb{R}^{n}\right) ;\langle\cdot\rangle^{s} \widehat{f} \in L^{2}\left(\mathbb{R}^{n}\right)\right\} .
$$

The norm in $H^{s}\left(\mathbb{R}^{n}\right)$ is given by

$$
\|f\|_{H^{s}\left(\mathbb{R}^{n}\right)}=\left\|\langle\cdot\rangle^{s} \widehat{f}\right\|_{L^{2}\left(\mathbb{R}^{n}\right)}
$$

and we may note that

$$
H^{-\infty}\left(\mathbb{R}^{n}\right)=\bigcup_{s \in \mathbb{R}} H^{s}\left(\mathbb{R}^{n}\right) \text { and } H^{\infty}\left(\mathbb{R}^{n}\right)=\bigcap_{s \in \mathbb{R}} H^{s}\left(\mathbb{R}^{n}\right) .
$$

We shall need the following properties:

$$
\forall s \in \mathbb{R} \text { the dual space of } H^{s}\left(\mathbb{R}^{n}\right) \text { is } H^{-s}\left(\mathbb{R}^{n}\right),
$$

and if $s<t$ we have

$$
\varphi\left(\mathbb{R}^{n}\right) \subset H^{\infty}\left(\mathbb{R}^{n}\right) \subset H^{t}\left(\mathbb{R}^{n}\right) \subset H^{s}\left(\mathbb{R}^{n}\right) \subset H^{-\infty}\left(\mathbb{R}^{n}\right) \subset \varphi^{\prime}\left(\mathbb{R}^{n}\right) .
$$

We recall the Littlewood-Paley decomposition [3].

Let $B(0, r)$ be the ball with radius $r$ in $\mathbb{R}^{n}$ and $\psi \in \mathcal{C}_{0}^{\infty}(B(0,2))$ such that $0 \leq \psi \leq 1$ and $\psi \equiv 1$ on $B(0,1)$. We have in $\mathrm{e}^{\infty}\left(\mathbb{R}^{n}\right)$ :

$$
\forall \xi \in \mathbb{R}^{n}: \lim _{N \rightarrow+\infty} \psi\left(2^{-N} \xi\right)=1
$$


Set $\chi(\xi)=\psi\left(2^{-1} \xi\right)-\psi(\xi), \chi_{k}(\xi)=\chi\left(2^{-k} \xi\right)$. Then we have:

$$
\lim _{N \rightarrow+\infty} \psi\left(2^{-N} \xi\right)=\psi(\xi)+\sum_{k=0}^{\infty} \chi_{k}(\xi) .
$$

For any $k=-1,0, \ldots$, and $u \in \varphi^{\prime}\left(\mathbb{R}^{n}\right)$, define the operator $\Delta_{k}$ as follows:

$$
\mathscr{F}\left(\Delta_{k} u\right)(\xi)=\chi_{k}(\xi) \widehat{u}(\xi), \quad k \geq 0
$$

and

$$
\left.\mathcal{F}_{\left(\Delta_{-1}\right.} u\right)(\xi)=\psi(\xi) \widehat{u}(\xi)
$$

It will be helpful in the following to have a representation of $\Delta_{k} u$ in the temporal space rather than in the frequential space. To that aim, we denote $\widehat{\phi}(\xi)=\chi(\xi)$ and $\phi_{k}(t)=2^{k n} \phi\left(2^{k} t\right)$ for $k \geq 0$.

Thus, $\widehat{\phi}_{k}(\xi)=\chi_{k}(\xi)$ and $\Delta_{k} u=\phi_{k} * u$ for $k \geq 0, \Delta_{-1} u=\mathscr{F}^{-1}(\psi) * u$. We note that $\Delta_{k} u \in \mathrm{C}^{\infty}\left(\mathbb{R}^{n}\right)$.

From (2) we obtain the Littlewood-Paley decomposition:

$$
u=\sum_{k=-1}^{\infty} \Delta_{k} u \text {. }
$$

Sobolev spaces can be redefined by means of $\Delta_{k}$ as follows:

$$
H^{s}\left(\mathbb{R}^{n}\right)=\left\{u \in \varphi^{\prime}\left(\mathbb{R}^{n}\right) ; \exists\left(c_{k}\right) \in l^{2}, \forall k \geq-1,\left\|\Delta_{k} u\right\|_{L^{2}\left(\mathbb{R}^{n}\right)} \leq c_{k} 2^{-k s}\right\} .
$$

Moreover, the norms $\|u\|_{H^{s}\left(\mathbb{R}^{n}\right)}$ and $\left\|2^{k s}\right\| \Delta_{k} u\left\|_{L^{2}\left(\mathbb{R}^{n}\right)}\right\|_{l^{2}}$ are equivalent.

The Besov space $B_{p, q}^{s}\left(\mathbb{R}^{n}\right), s \in \mathbb{R}, 1 \leq p \leq+\infty, 1 \leq q \leq+\infty$ is defined by

$$
B_{p, q}^{s}\left(\mathbb{R}^{n}\right)=\left\{u \in \varphi^{\prime}\left(\mathbb{R}^{n}\right) ; \exists\left(c_{k}\right) \in l^{q}, \forall k \geq-1,\left\|\Delta_{k} u\right\|_{L^{p}\left(\mathbb{R}^{n}\right)} \leq c_{k} 2^{-k s}\right\} .
$$

We set

$$
\|u\|_{B_{p, q}^{s}\left(\mathbb{R}^{n}\right)}=\left\|2^{k s}\right\| \Delta_{k} u\left\|_{L^{p}\left(\mathbb{R}^{n}\right)}\right\|_{l^{q}} .
$$

For $0<\rho<1$, the space $B_{\infty, \infty}^{\rho}\left(\mathbb{R}^{n}\right)$ is identical to the Hölder space

$$
C^{\rho}\left(\mathbb{R}^{n}\right)=\left\{u \in L^{\infty}\left(\mathbb{R}^{n}\right) ;|u(t)-u(s)| \leq C|t-s|^{\rho}\right\}
$$

By analogy with $C^{s}\left(\mathbb{R}^{n}\right)$, we introduce the distribution process space $C^{s}\left(\mathbb{R}^{n}, L^{2}(\Omega)\right)$ in the following way.

Let $X$ be a distribution process in $\varphi^{\prime}\left(\mathbb{R}^{n}, L^{2}(\Omega)\right)$. Then we define $\Delta_{k} X$ by duality:

$$
\left\langle\Delta_{k} X ; \varphi\right\rangle=\left\langle X ; \Delta_{k} \varphi\right\rangle=\left\langle X ; \phi_{k} * \varphi\right\rangle
$$

and

$$
\left\langle\Delta_{k} X ; \varphi\right\rangle=\left\langle\phi_{k} * X ; \varphi\right\rangle=\left\langle\phi_{k} * X, \varphi\right\rangle
$$


Thus

$$
\Delta_{k} X=\phi_{k} * X
$$

and in the same way,

For all $t \in \mathbb{R}^{n}$, we have

$$
\Delta_{-1} X=\mathscr{F}^{-1}(\psi) * X
$$

$$
\left\langle\Delta_{k} X ; \delta_{t}\right\rangle=\left\langle X ; 2^{k n} \phi\left(2^{k}(x-t)\right)\right\rangle=\Delta_{k} X(t) \in \mathrm{e}^{\infty}\left(\mathbb{R}^{n}\right), \text { for } k \geq 0 .
$$

Hence, $\Delta_{k} X \in \mathrm{C}^{\infty}\left(\mathbb{R}^{n} ; L^{2}(\Omega)\right)$.

For $s \in \mathbb{R}$, we set

$$
\begin{aligned}
& C^{s}\left(\mathbb{R}^{n}, L^{2}(\Omega)\right)= \\
& \quad\left\{X \in \mathcal{\varphi}^{\prime}\left(\mathbb{R}^{n}, L^{2}(\Omega)\right) ; \exists C, \forall k \geq-1,\|\| \Delta_{k} X\left\|_{L^{2}(\Omega)}\right\|_{L^{\infty}\left(\mathbb{R}^{n}\right)} \leq C 2^{-k s}\right\} .
\end{aligned}
$$

We can extend identity (3) to distribution process spaces by means of the following proposition.

Proposition 3: Let $X$ be a distribution process in $\varphi^{\prime}\left(\mathbb{R}^{n}, L^{2}(\Omega)\right)$ and $\rho \in(0 ; 1)$. $X$ belongs to $C^{\rho}\left(\mathbb{R}^{n}, L^{2}(\Omega)\right)$ if and only if $X$ is a process in $L^{\infty}\left(\mathbb{R}^{n}, L^{2}(\Omega)\right)$ and if there exists a constant $C$ such that for all $x, t \in \mathbb{R}^{n}$,

$$
\left\|X_{t+x}-X_{t}\right\|_{L^{2}(\Omega)} \leq C|x|^{\rho} .
$$

Proof: From now on all constants will be denoted by $C$. Assume that

$$
X \in L^{\infty}\left(\mathbb{R}^{n} ; L^{n}(\Omega)\right) \text { and }\left\|X_{t+x}-X_{t}\right\|_{L^{2}(\Omega)} \leq C|x|^{\rho} .
$$
It is clear that $\left\|\Delta_{-1} X_{t}\right\|_{L^{2}(\Omega)}$ is bounded. If $k \geq 0$, from $\chi(0)=0$ and $\int \phi(s) d s=$
0, we have

$$
\Delta_{k} X_{t}=\int 2^{k n}\left(X_{t-s}-X_{t}\right) \phi\left(2^{k} s\right) d s
$$

Hence,

$$
\begin{aligned}
\left(E\left(\Delta_{k} X_{t}\right)^{2}\right)^{1 / 2} & \leq \int 2^{k n} E\left(\left(X_{t-s}-X_{t}\right)^{2}\right)^{1 / 2}\left|\phi\left(2^{k} s\right)\right| d s \\
& \leq C \int 2^{k n}|s|^{\rho}\left|\phi\left(2^{k} s\right)\right| d s \\
& \leq C 2^{-k \rho} \int|x|^{\rho}|\phi(x)| d x
\end{aligned}
$$

Since $\phi \in \varphi\left(\mathbb{R}^{n}\right)$, this integral is finite.

So, $\forall k \geq-1$ we have \|\|$\Delta_{k} X\left\|_{L^{2}(\Omega)}\right\|_{L^{\infty}\left(\mathbb{R}^{n)}\right.} \leq C 2^{-k s}$ and $X \in$ $C^{\rho}\left(\mathbb{R}^{n}, L^{2}(\Omega)\right)$. Conversely, assume that $X \in C^{\rho}\left(\mathbb{R}^{n}, L^{2}(\Omega)\right)$. Hence, for all $k \geq-1$, 


$$
\begin{aligned}
& \sup _{t \in \mathbb{R}^{n}}\left\|\Delta_{k} X_{t}\right\|_{L^{2}(\Omega)} \leq C 2^{-k \rho} \text {. Since } \\
& \qquad X_{t}=\sum_{k=-1}^{+\infty} \Delta_{k} X_{t}
\end{aligned}
$$

and

then $X \in L^{\infty}\left(\mathbb{R}^{n} ; L^{2}(\Omega)\right)$. We have

$$
\left\|X_{t}\right\|_{L^{2}(\Omega)} \leq \sum_{k=-1}^{+\infty}\left\|\Delta_{k} X_{t}\right\|_{L^{2}(\Omega)} \leq C
$$

$$
X_{t}-X_{s}=\sum_{k=-1}^{\infty}\left(\Delta_{k} X_{t}-\Delta_{k} X_{s}\right)
$$

If $|t-s| \geq 1$, then

$$
\left\|X_{t}-X_{s}\right\|_{L^{2}(\Omega)} \leq 2 \sum_{k=-1}^{\infty}\left\|\Delta_{k} X_{t}\right\|_{L^{2}(\Omega)} \leq 2 \sum_{k=-1}^{\infty} C 2^{-k \rho} \leq C|t-s|^{\rho} .
$$

If $|t-s|<1$, choose $N \in \mathbb{N}$ such that

$$
2^{-N-1} \leq|t-s|<2^{-N} .
$$

Then,

$$
\left\|X_{t}-X_{s}\right\|_{L^{2}(\Omega)} \leq \sum_{k=-1}^{N-1}\left\|\Delta_{k} X_{t}-\Delta_{k} X_{s}\right\|_{L^{2}(\Omega)}+2 \sum_{k=N}^{\infty}\left\|\Delta_{k} X_{t}\right\|_{L^{2}(\Omega)}
$$

Now we have that

with

$$
\Delta_{k} X_{t}-\Delta_{k} X_{s}=(t-s) \int_{0}^{1} \partial\left(\Delta_{k} X\right)(\alpha s+(1-\alpha) t) d \alpha
$$

This leads to

$$
\partial \Delta_{k} X=\partial\left(\phi_{k} * X\right)=\partial \phi_{k} * X=\sum_{j=-1}^{\infty} \partial \phi_{k} * \Delta_{j} X=\sum_{j=-1}^{\infty} \phi_{j} * \partial \phi_{k} * X .
$$

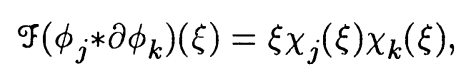

if $j \notin\{k-1, k, k+1\}, \chi_{j}(\xi) \chi_{k}(\xi) \equiv 0$ and $\phi_{j} * \partial \phi_{k} \equiv 0$, and, therefore,

$$
\partial \Delta_{k} X=\sum_{j=k-1}^{k+1} \phi_{j} * \partial \phi_{k} * X .
$$

Consequently,

$$
\begin{gathered}
\left\|\partial\left(\Delta_{k} X\right)\right\|_{L^{2}(\Omega)} \leq C \sum_{j=k-1}^{k+1}\left\|\partial \phi_{k} * \Delta_{j} X\right\|_{L^{2}(\Omega)} \\
\leq C \sum_{j=k-1}^{k+1}\left\|\partial \phi_{k}\right\|_{L^{1}(\mathbb{R})}\left\|\Delta_{j} X\right\|_{L^{2}(\Omega)} \leq C 2^{k(1-\rho)}
\end{gathered}
$$

because $\left\|\partial \phi_{k}\right\|_{L^{1}\left(\mathbb{R}^{n}\right)} \leq C 2^{k}$. It follows that 


$$
\begin{gathered}
\left\|\Delta_{k} X_{t}-\Delta_{k} X_{s}\right\|_{L^{2}(\Omega)} \leq|t-s| \int_{0}^{1}\left\|\partial\left(\Delta_{k} X\right)(\alpha s+(1-\alpha) t)\right\|_{L^{2}(\Omega)} d \alpha \\
\leq C|t-s| 2^{k(1-\rho)} .
\end{gathered}
$$

Hence,

$$
\begin{gathered}
\left\|X_{t}-X_{s}\right\|_{L^{2}(\Omega)} \leq C \sum_{k=-1}^{N-1}|t-s| 2^{k(1-\rho)}+2 C \sum_{k=N}^{\infty} 2^{-k \rho} \\
\leq C_{1}|t-s| 2^{N(1-\rho)}+C_{2} 2^{-N \rho} \leq C|t-s|^{\rho}
\end{gathered}
$$

because $2^{-N-1} \leq|t-s| \leq 2^{-N}$.

\section{Linear Distribution Process}

We now investigate processes that generalize linear Gaussian processes

$$
X_{t}=\int f(t-s) d W_{s}
$$

where $W_{s}$ is the standard Brownian motion and $f$ is a distribution.

\subsection{Definition of the process}

We shall write $\check{f}(t)=f(-t)$ if $f$ is a function and $\langle\check{f} ; \varphi\rangle=\langle f ; \check{\varphi}\rangle$ if $f$ is a distribution.

If $X_{t}=\int f(t-s) d W_{s}$ with $f \in \mathrm{E}^{2}\left(\mathbb{R}^{n}\right)$, then $X_{t}$ is a distribution process and for $\varphi \in \mathrm{C}_{0}^{\infty}\left(\mathbb{R}^{n}\right)$ we have $\langle X ; \varphi\rangle=\int\left(\int f(t-s) d W_{s}\right) \varphi(t) d t$. It is easy to see that in this case we can permute the integrals to obtain

$$
\forall \varphi \in \mathrm{C}_{0}^{\infty}\left(\mathbb{R}^{n}\right), \int\left(\int f(t-s) d W_{s}\right) \varphi(t) d t=\int\left(\int f(t-s) \varphi(t) d t\right) d W_{s} .
$$

Therefore, it is natural to introduce the following process:

$$
\langle X ; \varphi\rangle=\int \check{f} * \varphi(s) d W_{s}
$$

for $f \in \mathscr{D}^{\prime}\left(\mathbb{R}^{n}\right)$ such that $\check{f} * \varphi(s) \in L^{2}\left(\mathbb{R}^{n}\right)$ for all $\varphi \in \mathbb{C}_{0}^{\infty}\left(\mathbb{R}^{n}\right)$. Let us show that this is actually a distribution process.

Proposition 4: Let $f \in \mathscr{D}^{\prime}\left(\mathbb{R}^{n}\right)$ such that $\check{f} * \varphi(s) \in L^{2}\left(\mathbb{R}^{n}\right)$ for all $\varphi \in \mathbb{C}_{0}^{\infty}\left(\mathbb{R}^{n}\right)$. Let $W_{s}$ be the standard Brownian motion. The process defined by

$$
\langle X ; \varphi\rangle=\int \check{f} * \varphi(s) d W_{s}
$$

is a distribution process.

We call these processes, linear distribution processes with filter $f$.

Lemma 5: Let $f \in \mathscr{D}^{\prime}\left(\mathbb{R}^{n}\right)$ such that $\check{f} * \varphi(s) \in L^{2}\left(\mathbb{R}^{n}\right)$ for all $\varphi \in \mathbb{C}_{0}^{\infty}\left(\mathbb{R}^{n}\right)$. Then the application 


$$
\begin{gathered}
H: \mathrm{e}_{0}^{\infty}(\mathbb{R}) \rightarrow L^{2}\left(\mathbb{R}^{n}\right) \\
\varphi \mapsto \check{f} * \varphi
\end{gathered}
$$

is a linear continuous application.

Proof: Assume that the lemma holds true. For every compact set $K$ we have that:

$\exists C_{K}$ and $k \in \mathbb{N}, \forall \varphi \in \mathcal{C}_{0}^{\infty}(K)$, such that

$$
\begin{gathered}
\|\langle X ; \varphi\rangle\|_{L^{2}(\Omega)}=\|\check{f} * \varphi\|_{L^{2}\left(\mathbb{R}^{n}\right)} \\
\leq C_{K} \sup _{\substack{\alpha \mid \leq k \leq k \\
x \in \bar{K}}}\left|\partial_{x}^{\alpha} \varphi\right| .
\end{gathered}
$$

Hence, the linear application $X$ from $e_{0}^{\infty}\left(\mathbb{R}^{n}\right)$ into $L^{2}\left(\mathbb{R}^{n}\right)$ is continuous and is a distribution process.

Proof of the Lemma: In order to show that $H$ is continuous, we first show that the restriction $H_{\mid K}$ of $H$ to $e_{0}^{\infty}(K)$, where $K$ is a compact, is continuous.

Let $y \in \mathbb{R}^{n}$ be such that $|y| \leq R$. Since $f \in \mathscr{D}^{\prime}\left(\mathbb{R}^{n}\right)$, there exist $C_{K, R}$ and $k \in \mathbb{N}$, such that

$$
\begin{gathered}
\sup _{y \leq R}\left|\left\langle f ; \tau_{-y} \varphi\right\rangle\right| \leq C_{K, R} \sup _{\substack{|\alpha| \leq k \\
x \in K+B(0, R)}}\left|\partial_{x}^{\alpha}\left(\tau_{-y} \varphi(x)\right)\right| \\
\leq C_{K, R} \sup _{\substack{\alpha \mid \leq k \\
x \in K}}\left|\partial_{x}^{\alpha} \varphi(x)\right| .
\end{gathered}
$$

Therefore, $H_{\mid K}$ is continuous from $C_{0}^{\infty}(K)$ into $L_{l o c}^{\infty}\left(\mathbb{R}^{n}\right)$ and, by assumption, the range of $H_{\mid K}$ is included in $L^{2}\left(\mathbb{R}^{n}\right)$.

Since $e_{0}^{\infty}(K)$ and $L^{2}\left(\mathbb{R}^{n}\right)$ are complete and metrizable topological vector spaces, it suffices to use the closed graph theorem [23].

Let $\left(\varphi_{n}, f_{n}\right)$ be a convergent series in the graph of $H \mid K$. Then we have $f_{n}=$ $H_{\mid K}\left(\varphi_{n}\right)$ and $\left(\varphi_{n}, f_{n}\right)$ converges to $(\varphi, f)$ with respect to the $\mathrm{C}_{0}^{\infty}(K) \times L^{2}\left(\mathbb{R}^{n}\right)$ topology. Let us prove that $(\varphi, f)$ belongs to the graph. Since $H_{\mid K}$ is continuous from $\mathcal{C}_{0}^{\infty}(K)$ into $L_{\text {loc }}^{\infty}\left(\mathbb{R}^{n}\right)$, it follows that $H_{\left.\right|_{K}}\left(\varphi_{n}\right)$ converges to $H_{\mid K}(\varphi)$ in $L_{\text {loc }}^{\infty}\left(\mathbb{R}^{n}\right)$. Hence $f_{n}$ converges to $H_{\mid K}(\varphi)$ in $L_{l o c}^{\infty}\left(\mathbb{R}^{n}\right)$ and to $f$ in $L^{2}\left(\mathbb{R}^{n}\right)$. Since $L_{l o c}^{\infty}\left(\mathbb{R}^{n}\right) \subset$ $\mathfrak{D}^{\prime}\left(\mathbb{R}^{n}\right)$ and $L^{2}\left(\mathbb{R}^{n}\right) \subset \mathfrak{D}^{\prime}\left(\mathbb{R}^{n}\right)$ we obtain

$$
H_{\mid K}(\varphi)=f \text { in } \mathscr{D}^{\prime}\left(\mathbb{R}^{n}\right)
$$

Therefore, $(\varphi, f)$ belongs to the graph of $H_{\mid K}$, which proves that the graph is closed and that $H_{\mid K}$ is continuous. Consequently, $H$ is continuous from $e_{0}^{\infty}\left(\mathbb{R}^{n}\right)$ to $L^{2}\left(\mathbb{R}^{n}\right)$.

Thus, for each compact set $K$ we have

$$
\exists C_{K} \text { and } k \in \mathbb{N}, \forall \varphi \in \mathcal{C}_{0}^{\infty}(K) \text { such that }\|\check{f} * \varphi\|_{L^{2}\left(\mathbb{R}^{n}\right)} \leq C_{k} \sup _{\substack{\alpha \mid \leq k \\ x \in K}}\left|\partial_{x}^{\alpha} \varphi\right| \text {. }
$$


This inequality remains valid if $\varphi \in \mathrm{C}_{0}^{k}(K)$, as it can be seen by using standard arguments.

\subsection{Filters of linear distribution processes}

We will now characterize the distribution filters $f$ in $\mathscr{D}^{\prime}\left(\mathbb{R}^{n}\right)$ with which we can associate a distribution process.

Theorem 6: Consider $f \in \mathscr{I}^{\prime}\left(\mathbb{R}^{n}\right)$, then $\check{f} * \varphi \in L^{2}\left(\mathbb{R}^{n}\right)$ for all $\varphi \in \mathbb{C}_{0}^{\infty}\left(\mathbb{R}^{n}\right)$ if and only if $f \in H^{-\infty}\left(\mathbb{R}^{n}\right)$.

Proof: If $f \in H^{-\infty}\left(\mathbb{R}^{n}\right)$, then there exists $s \in \mathbb{R}$, such that $f \in H^{s}\left(\mathbb{R}^{n}\right)$. Let $\varphi$ be in $\mathcal{C}_{0}^{\infty}\left(\mathbb{R}^{n}\right)$. Using convolution properties it follows that $\breve{f} * \varphi \in \varphi^{\prime}\left(\mathbb{R}^{n}\right) \cap e^{\infty}\left(\mathbb{R}^{n}\right)$. Hence,

$$
\begin{aligned}
& \int|\widehat{f} * \varphi(x)|^{2} d x=\int|\mathcal{F}(\check{f} * \varphi)(\xi)|^{2} d \xi \\
& =\int\langle\xi\rangle^{2 s}|\mathcal{F}(\check{f})(\xi)|^{2} \frac{|\mathcal{F}(\varphi)(\xi)|^{2}}{\langle\xi\rangle^{2 s}} d \xi .
\end{aligned}
$$
Since $\mathscr{F}(\varphi) \in \varphi\left(\mathbb{R}^{n}\right)$ and $\langle\cdot\rangle^{s}|\mathcal{F}(\widehat{f})| \in L^{2}\left(\mathbb{R}^{n}\right), \frac{|\mathcal{F}(\varphi)(\xi)|^{2}}{\langle\xi\rangle^{2 s}}$ is bounded and therefore
$\widehat{f} * \varphi \in L^{2}\left(\mathbb{R}^{n}\right)$.

Conversely, we will show that $f$ is a linear continuous form defined on $H^{N}\left(\mathbb{R}^{n}\right)$ for some $N \in \mathbb{N}$, then $f \in H^{-N}\left(\mathbb{R}^{n}\right)$.

Let $\psi$ be in $\mathrm{C}_{0}^{\infty}\left(\mathbb{R}^{n}\right)$. From (5) we have

$$
\left|\int\left\langle f ; \tau_{-x} \varphi\right\rangle \psi(x) d x\right| \leq C_{K} \sup _{\substack{\alpha \mid \leq \leq \leq k \\ x \in K}}\left|\partial_{x}^{\alpha} \varphi\right|\|\psi\| L_{L^{2}\left(\mathbb{R}^{n}\right)}
$$

For $f \in L^{2}\left(\mathbb{R}^{n}\right)$ we also have

$$
\int\left\langle f ; \tau_{-x} \varphi\right\rangle \psi(x) d x=\left\langle f ; \int \varphi(x+\cdot) \psi(x) d x\right\rangle .
$$

But $\int \varphi(x+\cdot) \psi(x) d x \in \mathcal{C}_{0}^{\infty}\left(\mathbb{R}^{n}\right)$ and from (5) the left-hand side is well defined as soon as $\varphi \in \mathcal{C}_{0}^{k}(K)$. Therefore, this equality remains true for $f \in \mathscr{D}^{\prime}\left(\mathbb{R}^{n}\right)$ and $\varphi \in \mathrm{e}_{0}^{k}(K)$. The aim here is to show that we can replace $\varphi(x+y)$ by $\delta_{x=-y}$ in $(7)$ and obtain estimations in norm $H^{N}$.

Let $M \in \mathbb{N}$ and $\phi$ be such that $\widehat{\phi}=\frac{1}{\langle\xi\rangle^{2 M}} ; \phi \in H^{2 M-n / 2-\epsilon}\left(\mathbb{R}^{n}\right)$ and $(1-\Delta)^{M} \phi=\delta$, but $\phi$ is not compactly supported.

Let $\chi, \tilde{\chi}$ be cut-off functions in $e_{0}^{\infty}(B(0,1))$ such that $\chi=\tilde{\chi}=1$ on $B(0,1 / 2)$ and $\chi \tilde{\chi}=\tilde{\chi}$.

We have

$$
\tilde{\chi}(1-\Delta)^{M}(\chi \phi)=\delta
$$

By permuting $\tilde{\chi}$ with derivatives we can write

$$
\sum_{|\alpha| \leq 2 M} \partial_{x}^{\alpha}\left(\tilde{\chi}_{\alpha} \phi\right)=\delta
$$


where the $\tilde{\chi}_{\alpha}$ are linear combinations of $\tilde{\chi}$ 's derivatives. $\tilde{\chi}_{\alpha} \phi \in \mathrm{C}_{0}^{k}(K)$, as soon as $2 M-n-\epsilon>k$ and we can replace $\varphi$ by $\tilde{\chi}_{\alpha} \phi$ and $\psi$ by $\left(-\partial_{x}\right)^{\alpha} \psi$ in (6). Taking $K=B(0,1)$ and summing over $\alpha$, we obtain

$$
\left|\sum_{|\alpha| \leq 2 M}\left\langle f_{y} ; \int\left(\tilde{\chi}_{\alpha} \phi\right)(x+y)\left(-\partial_{x}\right)^{\alpha} \psi(x) d x\right\rangle\right| \leq C\left\|(1-\Delta)^{M} \psi\right\|_{L^{2}\left(\mathbb{R}^{n}\right)} .
$$

Integrating by parts, it follows that

$$
\begin{gathered}
\sum_{|\alpha| \leq 2 M} \int\left(\tilde{\chi}_{\alpha} \phi\right)(x+y)\left(-\partial_{x}\right)^{\alpha} \psi(x) d x=\left\langle\sum_{|\alpha| \leq 2 M} \partial_{x}^{\alpha}\left(\tilde{\chi}_{\alpha} \phi\right)(x+y) ; \psi(x)\right\rangle \\
=\left\langle\delta_{x=-y} ; \psi(x)\right\rangle=\psi(-y),
\end{gathered}
$$

and, consequently,

$$
|\langle f ; \tilde{\psi}\rangle| \leq C\left\|(1-\Delta)^{M} \psi\right\|_{L^{2}\left(\mathbb{R}^{n}\right)}=C\|\psi\|_{H^{2 M}\left(\mathbb{R}^{n}\right)}
$$

for $\psi \in \mathrm{C}_{0}^{\infty}\left(\mathbb{R}^{n}\right)$. As $\mathrm{C}_{0}^{\infty}\left(\mathbb{R}^{n}\right)$ is dense in $H^{2 M}\left(\mathbb{R}^{n}\right)$, it follows that $f$ is a linear continuous form on $H^{2 M}\left(\mathbb{R}^{n}\right)$, and $f \in H^{-2 M}\left(\mathbb{R}^{n}\right)$, that is $f \in H^{-\infty}\left(\mathbb{R}^{n}\right)$.

Remark: If $f \in H^{-\infty}\left(\mathbb{R}^{n}\right)$, then it is easy to see that $\breve{f} * \varphi \in L^{2}\left(\mathbb{R}^{n}\right), \forall \varphi \in \varphi\left(\mathbb{R}^{n}\right)$. Therefore, the processes defined by (4) are in $\varphi^{\prime}\left(\mathbb{R}^{n}, L^{2}(\Omega)\right)$.

\subsection{Moments of linear distribution processes}

Let $X$ be a distribution process with filter $f \in H^{-\infty}\left(\mathbb{R}^{n}\right)$.

Property 7: $X$ is a Gaussian stationary distribution process with zero mean and spectral density $|\widehat{f}(\xi)|^{2}$, i.e.,

$$
B(\varphi, \psi)=E\left(X(\varphi) \overline{X(\psi))}=\int \widehat{\varphi}(\xi) \overline{\widehat{\psi}(\xi)}|\widehat{f}(\xi)|^{2} d \xi=\langle\sigma ; \varphi * \bar{\psi}\rangle\right.
$$

where $\sigma \in \varphi^{\prime}\left(\mathbb{R}^{n}\right)$, the covariance of $X$, is given by

$$
\sigma=\mathscr{F}^{-1}\left(|\widehat{f}|^{2}\right)
$$

Proof: From

$$
\langle X ; \varphi\rangle=\int \check{f} * \varphi(s) d W_{s}
$$

it follows immediately that $X$ is a Gaussian distribution process with zero mean [8].

Let $\varphi, \psi$ be in $e_{0}^{\infty}\left(\mathbb{R}^{n}\right)$, then straightforward calculations give

$$
\begin{gathered}
\operatorname{cov}\left(X\left(\tau_{-h} \varphi\right), X\left(\tau_{-h} \psi\right)\right)=\int \widehat{\varphi}(\xi) \overline{\widehat{\psi}(\xi)}|\widehat{f}(\xi)|^{2} d \xi \\
=\operatorname{cov}(X(\varphi) ; X(\psi)) .
\end{gathered}
$$

Therefore, $X$ is a stationary distribution process [8].

From (8) it follows that the spectral density of $X$ is $|\widehat{f}|^{2}$. Since $|\widehat{f}|^{2} \in \varphi^{\prime}\left(\mathbb{R}^{n}\right)$, we have 


$$
\sigma=\mathscr{F}^{-1}\left(|\widehat{f}|^{2}\right) \in \mathscr{\varphi}^{\prime}\left(\mathbb{R}^{n}\right)
$$

which yields

$$
B(\varphi, \psi)=\langle\sigma ; \varphi * \bar{\psi}\rangle
$$

\subsection{Regularity of linear distribution processes}

We can characterize the regularity of a process $X$ with filter $f \in H^{-\infty}\left(\mathbb{R}^{n}\right)$ by the regularity of $f$.

Proposition 8: Let $f$ be in $H^{-\infty}\left(\mathbb{R}^{n}\right)$. Then the distribution process $X$ with filter $f$ belongs to $C^{s}\left(\mathbb{R}^{n}, L^{2}(\Omega)\right)$ if and only if $f \in B_{2, \infty}^{s}\left(\mathbb{R}^{n}\right)$.

Remark: For all $\epsilon>0$, we clearly have

$$
B_{2, \infty}^{s+\epsilon}\left(\mathbb{R}^{n}\right) \subset B_{2,2}^{s}\left(\mathbb{R}^{n}\right) \subset B_{2, \infty}^{s}\left(\mathbb{R}^{n}\right)
$$

Since $B_{2,2}^{s}\left(\mathbb{R}^{n}\right)=H^{s}\left(\mathbb{R}^{n}\right)$, we have $H^{-}\left(\mathbb{R}^{n}\right)=\bigcup_{s \in \mathbb{R}} B_{2, \infty}^{s}\left(\mathbb{R}^{n}\right)$. From this last property, the corresponding distribution processes belong to

$$
\left.C^{-\infty}\left(\mathbb{R}^{n}, L^{2}, \Omega\right)\right)=\bigcup_{s \in \mathbb{R}} C^{s}\left(\mathbb{R}^{n}, L^{2}(\Omega)\right) .
$$

Proof: Let $f$ be in $B_{2, \infty}^{s}\left(\mathbb{R}^{n}\right)$ and $X$ be the distribution process with filter $f$. Then, for all $k \geq-1$, we have

$$
\left\langle\Delta_{k} X ; \varphi\right\rangle=\int \check{f} * \Delta_{k} \varphi(s) d W_{s} .
$$

On the other hand,

$$
\check{f} * \Delta_{k} \varphi=\Delta_{k} \check{f} * \varphi
$$

with $\Delta_{k} f \in L^{2}\left(\mathbb{R}^{n}\right)$. Thus

$$
\left(\Delta_{k} X\right)_{t}=\int \Delta_{k} f(t-s) d W_{s}
$$

and $\Delta_{k} X$ is the process with filter $\Delta_{k} f$. Consequently,

$$
\left\|\Delta_{k} X\right\|_{L^{2}(\Omega)}^{2}=\left\|\Delta_{k} f\right\|_{L^{2}\left(\mathbb{R}^{n}\right)}^{2}
$$
Therefore, $\forall k \geq-1:\left\|\Delta_{k} X\right\|_{L^{2}(\Omega)}^{2} \leq C 2^{-2 k s}$ and $X \in C^{s}\left(\mathbb{R}^{n}, L^{2}(\Omega)\right)$. Conversely,
if $X \in C^{s}\left(\mathbb{R}^{n}, L^{2}(\Omega)\right)$ then

$$
\left\|\Delta_{k} X\right\|_{L^{2}(\Omega)} \leq C 2^{-k s} .
$$

Since $f \in H^{-\infty}\left(\mathbb{R}^{n}\right), \Delta_{k} f \in L^{2}\left(\mathbb{R}^{n}\right)$ and equality (9) implies that

$$
\left\|\Delta_{k} f\right\|_{L^{2}\left(\mathbb{R}^{n}\right)} \leq C 2^{-k s}
$$

Consequently, $f \in B_{2, \infty}^{s}\left(\mathbb{R}^{n}\right)$. 


\subsection{Distribution processes with stationary increments}

We now shall treat with linear Gaussian distribution processes with stationary increments.

The temporal processes, defined by

$$
X_{t}=\int(f(t-s)-g(-s)) d W_{s}
$$

where $f$ and $g$ are $L^{2}\left(\mathbb{R}^{n}\right)$ functions, have stationary increments. Indeed, for any reals $\delta$ and $h$ :

$$
\Delta_{h} X(t)=X(t+h)-X(t)=\int \Delta_{h} f(t-s) d W_{s},
$$

and the $\Delta_{h} X(t+\delta)$ are identically distributed.

Fractional Brownian motions [15] belong to this family, if we set

$$
g(t)=f(t)=\frac{1}{\Gamma\left(H+\frac{1}{2}\right)} t^{H-\frac{1}{2}} \rrbracket_{t>0} .
$$

Let $X$ be the distribution process associated with $X_{t}$, then we have

$$
\begin{aligned}
\langle X ; \varphi\rangle & =\int\left(\int(f(t-s)-g(-s)) \varphi(t) d t\right) d W_{s} \\
& =\int\left(\widehat{f} * \varphi-\check{g} \int \varphi(t) d t\right) d W_{s} .
\end{aligned}
$$

Processes, defined by (10) for distributions $f$ and $g$ such that $f * \varphi-\check{g} \int \varphi d t$ is in $L^{2}\left(\mathbb{R}^{n}\right)$ for all $\varphi \in \mathcal{C}_{0}^{\infty}\left(\mathbb{R}^{n}\right)$, are process with stationary increments, that is, the process $\Delta_{h} X=\tau_{h} X-X$ is a stationary distribution process

$$
\begin{gathered}
\left\langle\Delta_{h} X ; \varphi\right\rangle=\left\langle X ; \tau_{-h} \varphi\right\rangle-\langle X ; \varphi\rangle \\
=\int \check{f} *\left(\tau_{-h} \varphi-\varphi\right) d W_{s},
\end{gathered}
$$

and the random variables $\left\langle\Delta_{h} X ; \tau_{\delta} \varphi\right\rangle$ are identically distributed.

The following theorem gives a necessary and sufficient condition on $f$ and $g$ to have the distribution process $X$ in (10) well defined.

Theorem 9: Let $f$ and $g$ be in $\mathfrak{D}^{\prime}\left(\mathbb{R}^{n}\right)$. Then, $\check{f} * \varphi-\check{g} \int \varphi d t \in L^{2}\left(\mathbb{R}^{n}\right)$ for all $\varphi \in$ $e_{0}^{\infty}\left(\mathbb{R}^{n}\right)$ if and only if $\forall j=1, \ldots, n: \partial_{t}, g \in H^{-1}\left(\mathbb{R}^{n}\right)$, and $f-g \in H^{-\infty}\left(\mathbb{R}^{n}\right)$.

Remark: If $g \in L^{2}\left(\mathbb{R}^{n}\right)$, then the assumptions of Theorem 9 are verified for all $f \in H^{-\infty}\left(\mathbb{R}^{n}\right)$ and the result is a simple consequence of Theorem 6 . Nevertheless, the assumption $\partial_{j} g \in H^{-1}\left(\mathbb{R}^{n}\right)$ is weaker than the assumption $g \in L^{2}\left(\mathbb{R}^{n}\right)$.

In the fractional Brownian motion case, we have $\partial_{t} g \in L^{2}([1 ;+\infty[)$ and $g \in$ $L^{2}([0,2])$, which yields that $\partial_{t} g \in H^{-1}(\mathbb{R})$.

Proof: We shall need the following lemma.

Lemma 10: Let $g \in \mathscr{I}^{\prime}\left(\mathbb{R}^{n}\right)$. If $\forall j=1, \ldots, n: \partial_{t_{j}} g \in H^{-1}\left(\mathbb{R}^{n}\right)$, then we have,

$$
\forall \varphi \in \mathcal{C}_{0}^{\infty}\left(\mathbb{R}^{n}\right): \quad \check{g} * \varphi-\check{g} \int \varphi(t) d t \in L^{2}\left(\mathbb{R}^{n}\right) .
$$


Let us first assume that the lemma holds true. Let

$$
H(\varphi)=\check{f} * \varphi-\check{g} \int \varphi(t) d t .
$$

We can write

$$
H(\varphi)=(\check{f}-\check{g}) * \varphi+\left(\check{g} * \varphi-\check{g} \int \varphi(t) d t\right) .
$$

In the sequel, we denote $\partial_{t_{j}} g=\partial_{j} g$. If $\forall j=1, \ldots, n: \partial_{j} g \in H^{-1}\left(\mathbb{R}^{n}\right)$, and if there exists $\rho$ such that $f-g \in H^{\rho}\left(\mathbb{R}^{n}\right)$, then the first term belongs to $L^{2}\left(\mathbb{R}^{n}\right)$ by Theorem 6 and the second term belongs to $L^{2}\left(\mathbb{R}^{n}\right)$ due to the lemma.

Conversely, assume that for all $\varphi \in \mathrm{C}_{0}^{\infty}\left(\mathbb{R}^{n}\right), H(\varphi) \in L^{2}\left(\mathbb{R}^{n}\right)$ and, therefore, $H\left(\partial_{j} \varphi\right) \in L^{2}\left(\mathbb{R}^{n}\right)$. Now, $H\left(\partial_{j} \varphi\right)=\partial_{j} \check{f} * \varphi$ and by Theorem 6 there exists a $\rho$ such that $\partial_{j} \check{f} \in H^{\rho}\left(\mathbb{R}^{n}\right)$. On the other hand, if $H(\varphi) \in L^{2}\left(\mathbb{R}^{n}\right)$, then $\partial_{j}(H(\varphi)) \in$ $H^{-1}\left(\mathbb{R}^{n}\right)$. Since

$$
\partial_{j}(H(\varphi))=\partial_{j}(\check{f}) * \varphi-\partial_{j}(\check{g}) \int \varphi(t) d t,
$$

it follows that $\partial_{j}(\check{g}) \int \varphi \in H^{-1}\left(\mathbb{R}^{n}\right)$. Let $\varphi$ be such that $\int \varphi(t) d t=1$. For any $j=1, \ldots, n$ we have $\partial_{j}(\check{g}) \in H^{-1}\left(\mathbb{R}^{n}\right)$.

Since,

$$
(\check{f}-\check{g}) * \varphi=H(\varphi)-\left(\check{g} * \varphi-\check{g} \int \varphi\right),
$$

where the second term is in $L^{2}\left(\mathbb{R}^{n}\right)$, due to the lemma, we have

$$
\forall \varphi \in C_{0}^{\infty}\left(\mathbb{R}^{n}\right): \quad(\check{f}-\check{g}) * \varphi \in L^{2}\left(\mathbb{R}^{n}\right),
$$

and by Theorem 6 there exists a $\rho$ such that $\check{f}-\check{g} \in H^{\rho}\left(\mathbb{R}^{n}\right)$.

Proof of the Lemma: If for any $j=1, \ldots n: \partial_{j} g \in H^{-1}\left(\mathbb{R}^{n}\right)$, then $g \in \Psi^{\prime}\left(\mathbb{R}^{n}\right)$ and

$$
\begin{gathered}
\left\|\check{g} * \varphi-\check{g} \int \varphi(t) d t\right\|_{L^{2}\left(\mathbb{R}^{n}\right)}=\int|\widehat{g}(-\xi)|^{2}|\widehat{\varphi}(\xi)-\widehat{\varphi}(0)|^{2} d \xi \\
\leq C \int\langle\xi\rangle^{-1}|\xi|^{2}|\widehat{g}(-\xi)|^{2} d \xi \\
\leq C \sum_{j=1}^{n} \int\langle\xi\rangle^{-2}\left|\partial_{j} \widehat{g}(\xi)\right|^{2} d \xi \\
\leq C \sum_{j=1}^{n}\left\|\partial_{j} g\right\|_{H^{-1}\left(\mathbb{R}^{n}\right)}<+\infty .
\end{gathered}
$$

Hence, $\check{g} * \varphi-\check{g} \int \varphi(t) \in L^{2}\left(\mathbb{R}^{n}\right)$.

Corollary 11: If $X$ is a process defined by (10), then the process $\partial_{j} X$ is a stationary linear distribution process with filter $\partial_{j} f$ and with spectral density $\left|\xi \widehat{f}\left(\xi_{j}\right)\right|^{2}$.

Proof: If $X$ is a distribution process defined by (10), then 


$$
\left\langle\partial_{j} X ; \varphi\right\rangle=-\left\langle X ; \partial_{j} \varphi\right\rangle=\int \check{\partial}_{j} f * \varphi d W_{s}
$$

According to Section 3.3, $\partial_{j} X$ is a stationary process with spectral density $\left|\partial_{j} \widehat{f}(\xi)\right|^{2}=\left|\xi_{j} \widehat{f}(\xi)\right|^{2}$.

Remark: In the fractional Brownian motion case we find the density of the fractional Gaussian noise [4] in the form:

$$
\left|\Gamma\left(H+\frac{1}{2}\right) t_{+}^{\widehat{H}-\frac{1}{2}}\right|^{2}=\left|\xi(i \xi)^{-H-\frac{1}{2}}\right|^{2}=|\xi|^{1-2 H} .
$$

Noting that the temporal process, defined by

$$
X_{t}=\int\left(f(t-s)-\sum_{|\alpha| \leq k-1} t^{\alpha} g_{\alpha}(-s)\right) d W_{s}
$$

has stationary $k$ th order increments, in the same way we can define distribution processes with stationary $k$ th order increments, as

$$
\langle X ; \varphi\rangle=\int\left(\check{f} * \varphi(s)-\sum_{|\alpha| \leq k-1} g_{\alpha}(-s) \int \varphi(t) t^{\alpha} d t\right) d W_{s} .
$$

The following result generalizes Theorem 9 .

Theorem 12: Let $f$ and $\left\{g_{\alpha}\right\}, \quad|\alpha| \leq k-1, \alpha \in \mathbb{N}^{n}$ be in $\mathscr{D}^{\prime}\left(\mathbb{R}^{n}\right)$. Then $\check{f} * \varphi(s)-g_{\alpha}(-s) \sum|\alpha| \leq k-1 \int \varphi(t) t^{\alpha} d t \in E^{2}\left(\mathbb{R}^{n}\right)$ for all $\varphi \in \mathcal{C}_{0}^{\infty}\left(\mathbb{R}^{n}\right)$, if and only if

$$
\begin{gathered}
\forall|\beta| \leq k-1 ; \partial^{\beta} g_{\alpha} \in H^{-|\beta|}\left(\mathbb{R}^{n}\right) \text { for }|\alpha|+|\beta|=k \\
\partial^{\beta} g_{0}-\beta ! g_{\beta} \in H^{-|\beta|}\left(\mathbb{R}^{n}\right) \text { and } f-g_{0} \in H^{-\infty}\left(\mathbb{R}^{n}\right) .
\end{gathered}
$$

The proof can be rendered by recurrence in the same way as that of Theorem 9 .

\section{References}

[1] Bel, L., Oppenheim, G., Robbiano, L. and Viano, M.C., Distribution processes of the fractional ARMA type, mixing properties, Prob. and Math. Statistics 16:2 (1996), 311-336.

[2] Brillinger, D.R., Asymptotic normality of finite Fourier transforms of stationary generalized processes, J. of Multivariate Analysis 12 (1982), 64-71.

[3] Coifman, E. and Meyer, Y., Au delà des Opérateurs Pseudo-Différentiels, Astérisque 57, Paris 1978.

[4] Drouilhet, R., Dérivée du Mouvement Brownien Fractionnaire et estimation de densité spectrale, Thèse, Université de Pau 1993.

[5] Esquivel, M.L., Sur une classe de distributions aléatoires, Ann. Sci. Math. Québec 17:2 (19y3), 169-186.

[6] Fernandez, B. and Gorostiza, L.G., A criterion of convergence of generalized processes and an application to a super critical branching particle system, Canad. J. of Math. 43:5 (1991), 985-987.

[7] Fernique, X., Processus linéaires, processus généralisés, Annales de l'Institut Fourier 67 (1967), 2-92. 
[8] Gelfand, I.M. and Vilenkin, N.Y., Generalized Functions Vol. 4, Academic Press, New York 1964.

[9] Hida, T., Functionals of Brownian Motion, Lectures in Applied Mathematics and Informatics, Manchester Univ. Press, Manchester 1990.

[10] Inaba, H. and Tapley, B.D., Generalized random processes: A theory and the white Gaussian process, SIAM J. of Control 13:3 (1975), 719-735.

[11] Itô, K., Stationary random distributions, Mem. Coll. Sci. Kyoto Univ. Ser A 28:3 (1954), 209-223.

[12] Itô, Y., Stationary Fourier hyperprocesses, Publ. Res. Inst. Math. Sci. 22:1 (1986), 31-42.

[13] Kuo, H.H., White Noise Calculus. Algebra, Analysis and Geometry, World Scientific Publishing, Teanek, N.J. 1989.

[14] Lozanov-Crvenković, Z. and Pilipović, S., Gaussian generalized random processes on $\mathscr{K}_{6}\left\{M_{p}\right\}$ spaces, J. of Math. Anal. and Appl. 181 (1994), 755-761.

[15] Mandelbrot, B.B. and Van Ness, J.W., Fractional Brownian motion, fractional noises and applications, SIAM Review 10:4 (1968), 422-437.

[16] Meidan, R., Reproducing kernel Hilbert spaces of distributions and generalized stochastic processes, SIAM J. on Math. Analysis 10:1 (1979), 62-70.

[17] Meidan, R., On the connection between ordinary and generalized stochastic processes, J. of Math. Anal. and Appl. 76 (1980), 124-133.

[18] Mitoma, I., On the sample continuity of $S^{\prime}$-processes, J. of the Math. Soc. of Japan 35:4 (1983), 629-636.

[19] Piterbag, L.I., On the structure of the last splitting $\sigma$-algebra for a generalized Gaussian field with a rational spectrum, Theory of Probability and its Applications 31:2 (1987), 351-355.

[20] Pugh, E.L., The generalized analytic signal, J. of Math. Anal. and Appl. 89 (1982), 674-699.

[21] Rao, M.M., Characterization and extension of generalized harmonizable random fields, Proc. Nat. Acad. of Science USA 58 (1967), 1213-1219.

[22] Schwartz, L, Théorie des Distributions, Hermann, Paris 1966.

[23] Trêves, F., Topological Vector Spaces, Distributions and Kernels, Academic Press, New York 1967.

[24] Triebel, H., Theory of Function Spaces, Birkhauser, Boston 1992. 


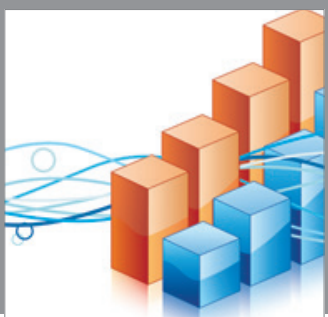

Advances in

Operations Research

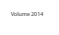

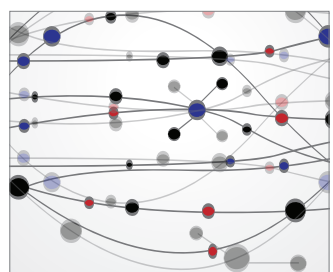

\section{The Scientific} World Journal
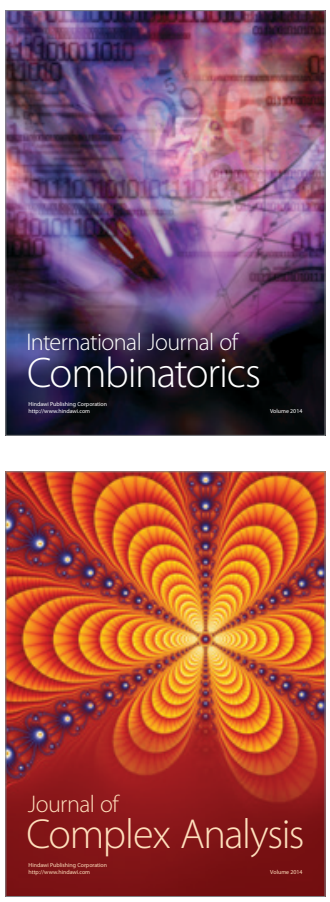

International Journal of

Mathematics and

Mathematical

Sciences
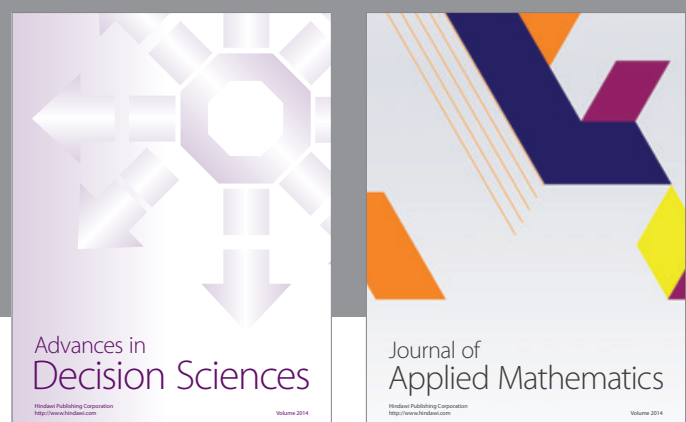

Journal of

Applied Mathematics
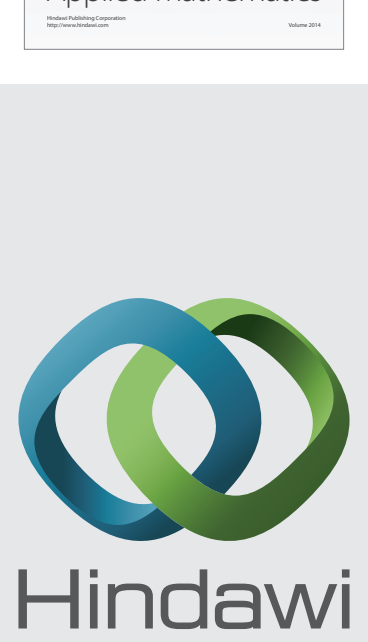

Submit your manuscripts at http://www.hindawi.com
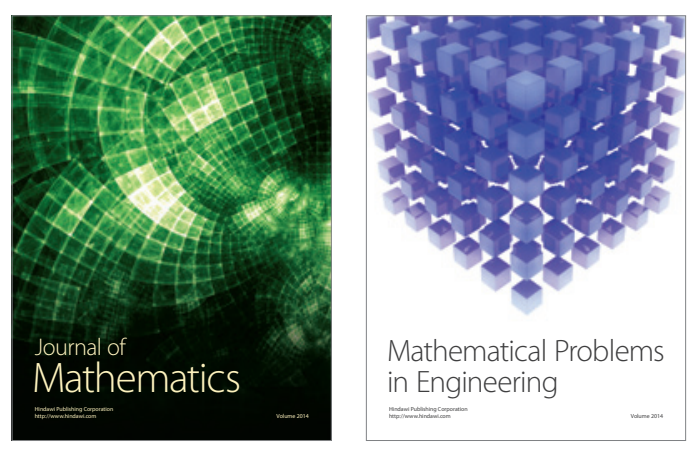

Mathematical Problems in Engineering
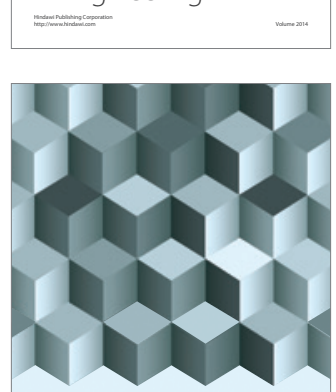

Journal of

Function Spaces
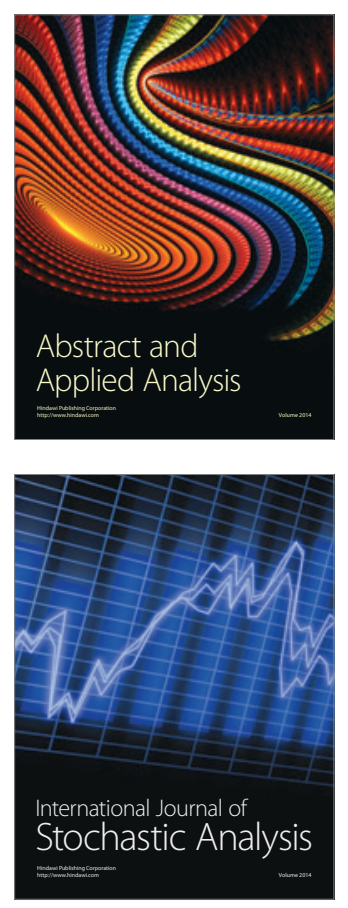

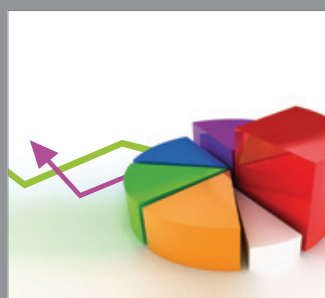

ournal of

Probability and Statistics

Promensencen
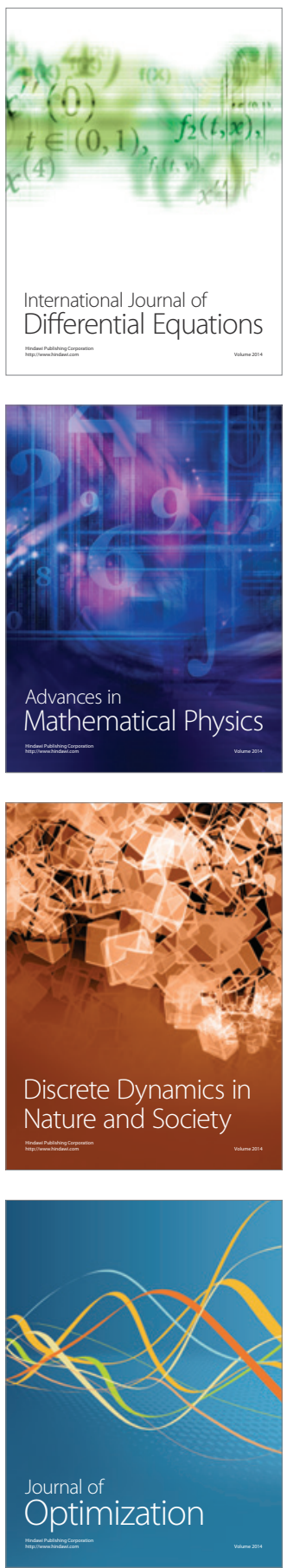